\title{
THE ULTRASTRUCTURE OF THE BRAIN OF ADULT LIVER FLUKE, Opisthorchis viverrini
}

\author{
LEKSOMBOON R. ${ }^{1}$, JONES M.K. ${ }^{2}$, CHAIJAROONKHANARAK W. ${ }^{3}$, CHAIWONG T. ${ }^{1}$, KHRONGYUT S. ${ }^{1}$ AND \\ SRIPA B. , $5 *^{5 *}$ \\ ${ }^{1}$ College of Medicine and Public Health Ubon Ratchathani University 34190, Thailand. \\ ${ }^{2}$ School of Veterinary Sciences, The University of Queensland, Brisbane, Queensland 4072, Australia \\ 3Department of Anatomy, Faculty of Medicine, Khon Kaen University, Khon Kaen 40002, Thailand \\ ${ }^{4}$ Tropical Disease Research Laboratory, Department of Pathology, Faculty of Medicine, Khon Kaen University, Khon Kaen 40002, Thailand \\ ${ }^{5}$ Liver fluke and Cholangiocarcinoma Research Center, Khon Kaen University, Khon Kaen 40002, Thailand \\ ${ }^{*}$ Corresponding Author: Email- banchob@kku.ac.th
}

Received: August 17, 2012; Accepted: August 24, 2012

\begin{abstract}
The organization of the nervous system has been documented for various helminth parasites. However, the ultrastructure of the brain of the carcinogenic liver fluke, Opisthorchis viverrini has not been described. This study therefore clarified the fine structure of the cerebral ganglia (brain) of this fluke using transmission electron microscopy. The cerebral ganglia of 0 . viverrini are composed of ganglion cell bodies and the bilateral lobe of neuropile with a tight packing of unmyelinated axon processes. The two lobes of the brain are connected together by transverse commissure. The neuropiles were insinuated by groups or bundles of giant axons and mesenchymal processes. Cells and axon processes of the 0 . viverrini brain were rich in tight accumulations of abundant neuronal mediator vesicles. Numerous synaptic contacts were present in the neuropile of brain and scattered in transverse commissure. The organization of the brain of this liver fluke brain was comparable to other trematodes.
\end{abstract}

Keywords- Opisthorchis viverrini, brain, cerebral ganglia, nervous system, ultrastucture, transmission electron microscope

Citation: Leksomboon R., et al. (2012) The Ultrastructure of the Brain of Adult Liver Fluke, Opisthorchis viverrini. International Journal of Parasitology Research, ISSN: 0975-3702 \& E-ISSN: 0975-9182, Volume 4, Issue 2, pp.-90-93.

Copyright: Copyright@2012 Leksomboon R., et al. This is an open-access article distributed under the terms of the Creative Commons Attribution License, which permits unrestricted use, distribution and reproduction in any medium, provided the original author and source are credited.

\section{Introduction}

Opisthorchis viverrini (O. viverrini) is a liver fluke belonging to Phylum Platyhelminthes, Class Trematoda, People become infected by consuming food containing uncooked fish contaminated with the metacercariae of this species. The metacercariae excyst in the duodenum, releasing juvenile 0 . viverrini that then migrate up into the bile ducts and develop to maturity. The worms must use their nervous systems to respond to some chemotactic agents. Adult worms reside in the bile duct and generate inflammation in the liver that contributes to the development of cholangiocarcinoma (CCA) which is one of the highest cause of death in Northeast Thailand [11]. The nervous system of $O$. viverrini and other trematode have been studied by immuno-histochemical methods with antibodies against different neurotransmitters $[6,7]$ while ultrastructural of the nervous system of this worm have never been reported. This study is aimed to clarify the ultrastructure of the cerebral ganglia of adult $O$. viverrini.

\section{Material and Methods}

The protocols are the routine techniques of The Centre for Microscopy and Microanalysis (CMM), of The University of Queensland. Worms were initially fixed in $3 \%$ glutaraldehyde in $0.1 \mathrm{M}$ sodium cacodylate. All processing proceeded using microwave irradiation (Biowave, PELCO) to expedite sample processing. Primary fixation proceeded under vacuum at a current of 150 watts for periods of $20 \mathrm{sec}$ on, $20 \mathrm{sec}$ off, $20 \mathrm{sec}$ on. This fixation protocol was repeated twice. Specimens were rinsed with $0.1 \mathrm{M}$ sodium cacodylate and ran in microwave for a power 80 watts time $40 \mathrm{sec}$ with vacuum. The specimens were then fixed under vacuum by reduced osmium ( $2 \%$ osmium tetroxide in $3 \%$ potassium ferricyanide) in microwave oven for a setting of 80 watts for 20 sec power on, 20 $\mathrm{sec}$ off, $20 \mathrm{sec}$ on and repeat (2 time). Water rinse was done in microwave for a power 80 watts time $40 \mathrm{sec}$ with vacuum on. $2 \%$ Uranyl acetate enbloc staining (Optional) in microwave for a power 150 watts time $60 \mathrm{sec}$ on, $60 \mathrm{sec}$ off, $60 \mathrm{sec}$ on and vacuum on. 
Dehydration was performed for $40 \%, 50 \%, 70 \%, 90 \%$, and 2 time $100 \%$ ethanol in microwave for a power 250 watts time $40 \mathrm{sec}$ with vacuum off. The samples were infiltrated with ascending concentrations of Epon 812 for 1:2, 1:1, 2:1, pure resin 2 times in ethanol) in microwave with a power of 250 watts and time 3 min under vacuum. The samples were immersed in $100 \%$ resin overnight prior to embedding in EPON resin. The processes after this follow conventional processing techniques. Ultrathin sections were cut at cerebral ganglia location which shown by light-microscopy [7].

\section{Results}

\section{Cerebral Ganglia and Neuropiles}

The cerebral ganglion is composed of ganglion cell bodies and the bilateral lobe of the central neuropile. Both cerebral ganglia were identified postero-dorsal to the pharynx and esophagus. The ganglion cell bodies were few in number. The neuropile was made of small and large unmyelinated axon processes. The couple of cerebral ganglion were connected each other by transverse commissure. The neuronal vesicles in the neuropile of the cerebral lobes appeared relatively more dominant than the transverse commissure region [Fig-1,2].

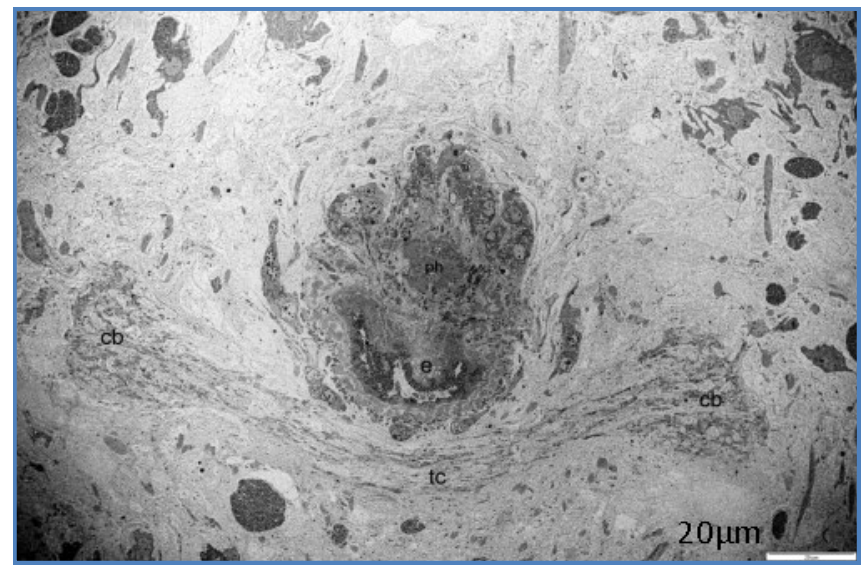

Fig. 1- Ultrastructure of cerebral ganglion of adult stage. Both cerebral ganglia (cb) were present posterodorsally to pharynx (ph) and the first part of esophagus (e). Two side of cerebral ganglion were connected by the transverse commissure (tc)

The neuropile of the cerebral ganglion consisted of a tight packing of unmyelinated nerve processes. The nerve processes were relatively irregular with regard to the arrangement, shape and size. Large diameter-processes termed giant processes and mesenchymal processes were seen in and surrounded the neuropile. The axon and giant nerve processes contained mediator vesicles, microtubules and mitochondria. However, the giant processes contain fewer vesicles when compared to most axon processes in the neuropile clusters. The giant nerve processes are intimately associated with each other, and their cell membranes often exhibited extensive invaginations. The mesenchyme processes themselves contain numerous darkly-staining mitochondria, and small granules [Fig-2].

\section{Vesicles}

Vesicles in nerve processes in the cerebral ganglia of 0 . viverrini are classified into 5 groups [Fig-2,3] (1) small, clear vesicles (sv) measuring about 10-30 nm in diameter, (2) dense-core vesicles (dcv) measuring about 70-120 nm in diameter, (3) large dense vesicles (Idv) measuring about 130-560 nm in diameter, (4) large lucent vesicles (llv) measuring about 40-100 nm in diameter, (5) small dense granules (sdg) measuring less than $10 \mathrm{~nm}$ in diameter [13].

\section{Synaptic Contacts}

Numerous synaptic contacts were present in the neuropile. The occurrences of synaptic contacts in the transverse commissure were less frequent than in the cerebral ganglia [Fig-2-5].

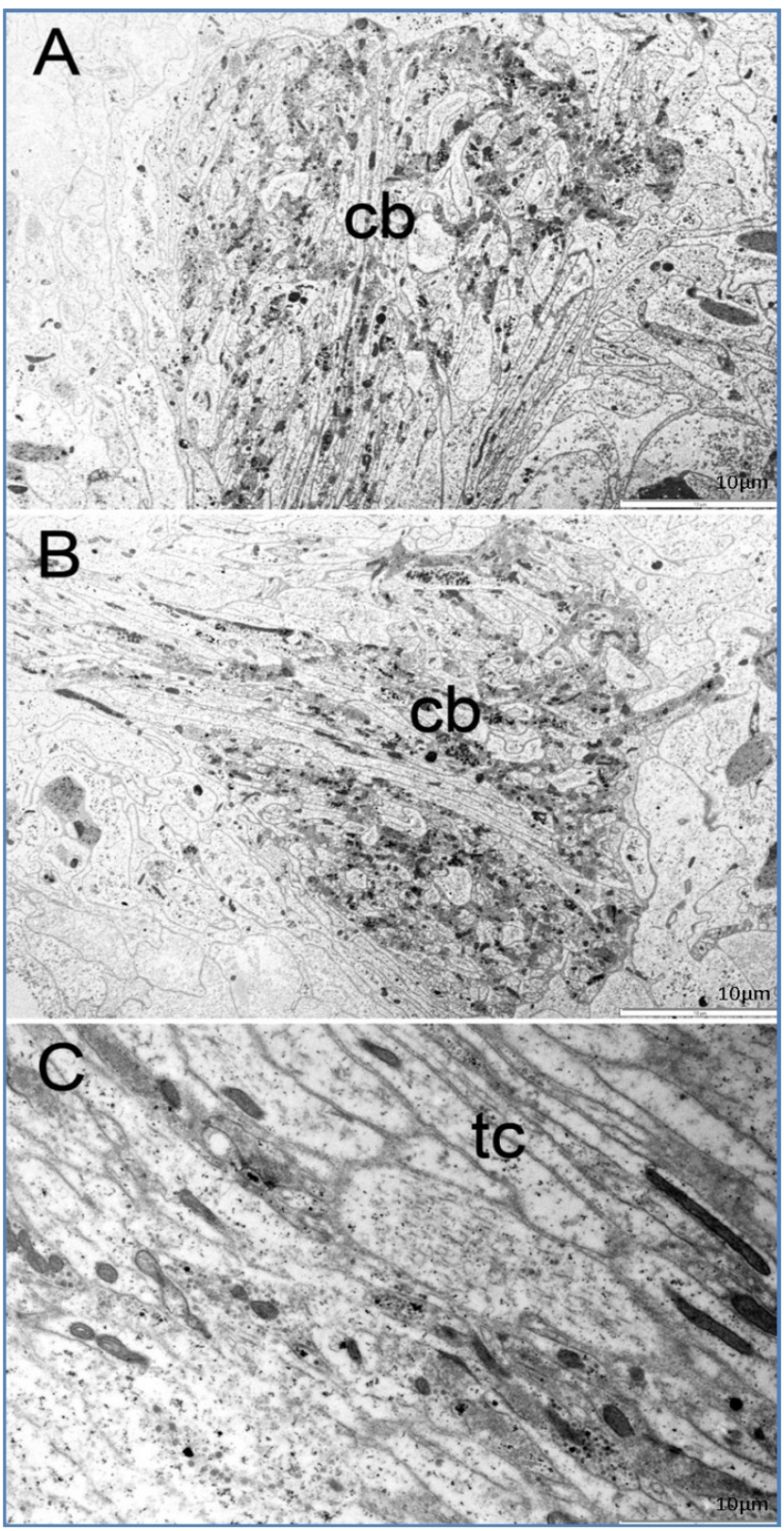

Fig. 2(A-C)- Ultrastructure of each side of cerebral ganglion (cb) of adult stage and a part of transverse comissure (tc). The cerebral ganglion contained neuropile and its was surrounded by mesenchymal processes and giant axon processes (pale area). A few ganglion cell bodies are located nearby. Some giant nerve cell processes and mesenchymal cells processes also found throughout the ganglion. 


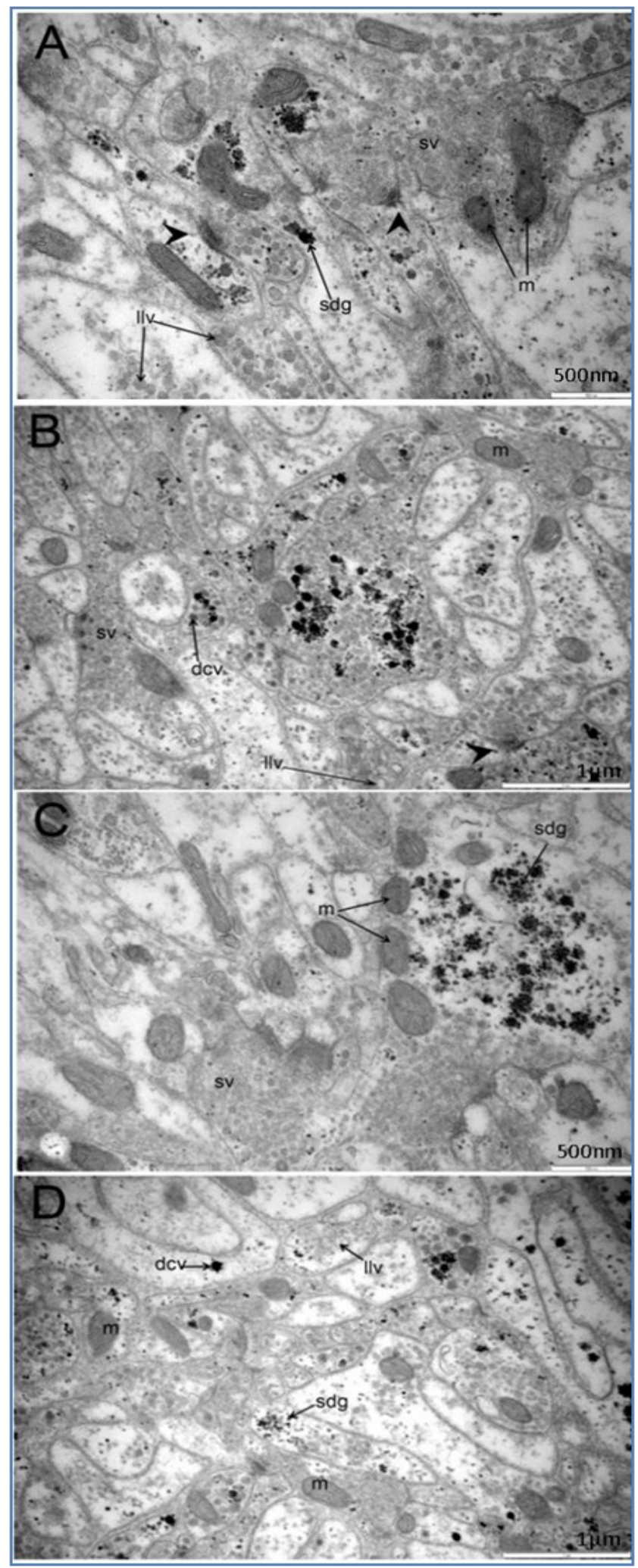

Fig. 3(A-D)- Ultrastructure of neuropiles of cerebral ganglion of adult stage. The neuropiles contained a number of mitochondria (m) and various type of neuronal vesicles including small clear vesicles (sv), dense core vesicles (dcv), large dense vesicles (Idv), large lucent vesicles (llv) and small dense granules (sdg). The synapses (arrow) were often found in general cerebral ganglion.
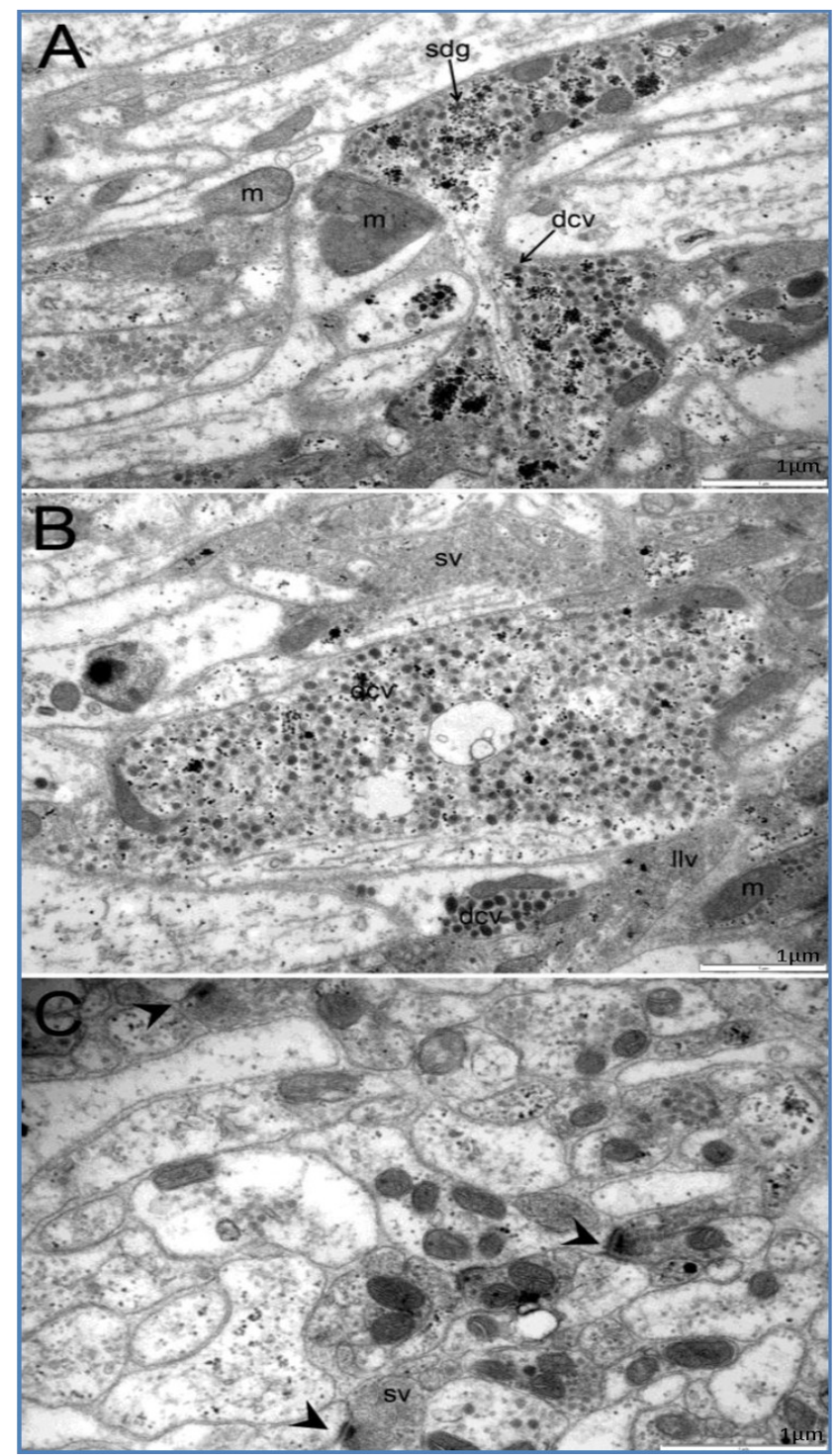

Fig. 4(A-C)- Ultrastructure of neuropiles of the cerebral ganglia (cb) at adult stage. The neuropiles contained mitochondria $(\mathrm{m})$ and neuronal vesicles of various types such as small clear vesicles (sv), dense-core vesicles (dcv), large dense vesicles (ldv), large lucent vesicles (llv) and small dense granules (sdg). Synapses (arrow) were often found throughout the cerebral ganglia

\section{Discussion}

\section{Cerebral Ganglia and Neuropiles}

O. viverrini cerebral ganglia consist of two lobes of neuropiles with a bundle of transverse fibers connection. Few ganglion cell bodies were scattered around the neuropile. These event in structures and morphologies of the cerebral ganglia are in accordance with the observations of several invertebrates including Fasciola hepatica [12], Schistosoma mansoni [4], Macrostomum lignano [9] and also resembles $S$. roscoffensis [2].

The phenomenon of the cerebral ganglia of $O$. viverrini by insinuation of giant processes and mesenchymal processes resembles the central ganglionic type brain of other flatworms $[2,4,8,12]$. These processes may be concerned in the provision of nutrients to the 
neurons [10]. Sukhdeo and Sukhdeo (1990) mentioned that the nervous system may require a number of giant nerve processes for increased size adaptation during development. Another postulate relates to the body movement in such soft-bodied animals. For example, $F$. hepatica stretches and contracts during movement, significantly altering its body length. This movement results in the stretching of nerve processes.

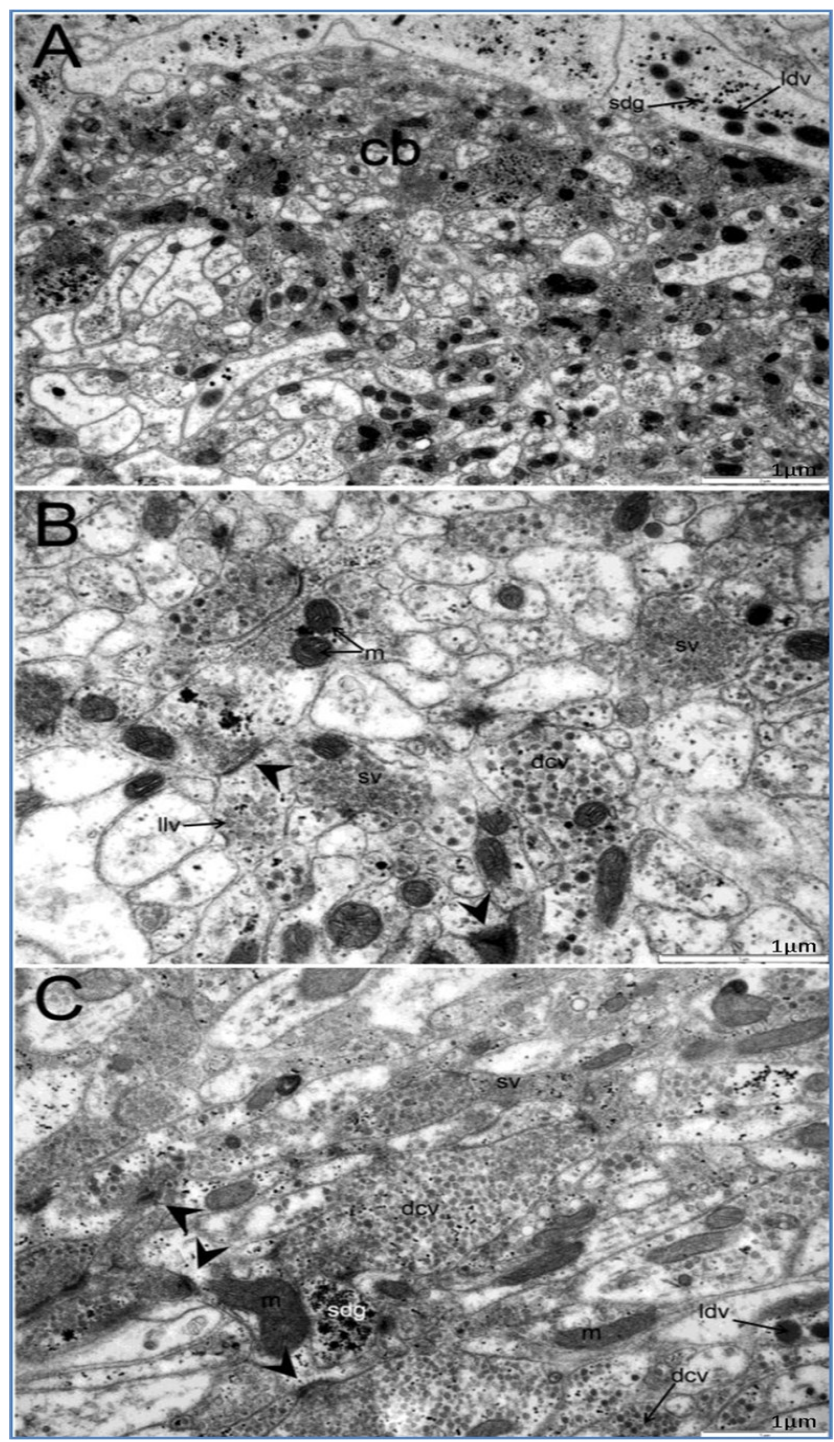

Fig. 5(A-C)- Ultrastructure of neuropiles of the cerebral ganglia (cb) at adult stage. The neuropiles contained mitochondrias $(\mathrm{m})$ and various types of neuronal vesicles including small clear vesicles (sv), dense-core vesicles (dcv), large, dense vesicles (ldv), large, lucent vesicles (llv) and small dense granules (sdg). Synapses (arrow) were often found in general cerebralganglion.

\section{Vesicles}

Cells and axon processes of the 0 . viverrini nervous system were rich in tight accumulations of abundant neuronal mediator vesicles of five types. The three most common types of vesicles (sv, dcv and Iv (large vesicular organelles) are also found in the nervous system of all phyla (e.g., Platyhelminths, Arthropoda, Vertebrata) [1]. The diversity in both size and structure of vesicles in the nervous system of flatworms were determined by different neuronal mediator vesicles. However, those classifications may be unreliable because they were not consistent with the results of immunogold labelling in electron microscope observations. The size and structure of vesicle probable depends on the developmental stages of worm [5].

\section{Synaptic Contact}

Numerous asymmetric (triad or wedge-shaped) and symmetric synapses were present in the neuropile of both lobes of the 0 . viverrini brain, similar to several trematode such as $F$. hepatica $[3,12]$. In $S$. roscoffensis, these synapses establish contact between the different neurons within central and peripheral nervous system [2]. In the classical literature in electron microscopy, excitatory transmission is a responsibility of asymmetric synapses while inhibitory is a responsibility of symmetric synapses transmission [2]. However, the correlations between function and morphology of synapses in the 0 . viverrini nervous system still requires further development.

\section{Acknowledgements}

Thank you for all staff of Department of Anatomy and the Tropical Disease Research, Department of Pathology, Faculty of Medicine, Khon Kaen University, Khon Kaen, Thailand. The Centre for Microscopy and Microanalysis (CMM) of University of Queensland, Brisbane, Queensland, Australia. This study was supported by College of Medicine and Public Health, Ubon Ratchathani University, The Graduate School Khon Kaen University and Faculty of Medicine Khon Kaen University, Thailand.

\section{References}

[1] Bedini C. and Lanfranchi A. (1991) Acta. Zool., 72, 101-106.

[2] Bery A., Cardona A., Martinez P., Hartenstein V. (2010) Dev. Genes Evol., 220, 61-76.

[3] Dixon K.E., Mercer E.H. (1965) J. Parasitol., 51, 967-976.

[4] Dorsey C.H., Cousin C.E., Lewis F.A., Stirewalt M.A. (2002) Micron., 33, 279-323.

[5] Halton D.W., Gustafsson M.K.S. (1996) Parasitology, 113, 4772.

[6] Kotikova E.A., Raikova O.I., Reuter M., Gustafsson M.K. (2002) Tissue Cell., 34, 365-374.

[7] Leksomboon R., Chaijaroonkhanarak W., Arunyanart C., Umka J., Jones M.K., Sripa B. (2011) Parasitol Int., 61, 107-111.

[8] Mori I., Sasakura H., Kuhara A. (2007) Curr. Opin. Neurobiol., 17, 712-719.

[9] Morris J., Cardona A., De Miguel-BonetMdel M., Hartenstein V. (2007) Dev. Genes Evol., 217, 569-584.

[10]Saint Marie R.L., Carlson S.D. (1983) J. Neurocy., 12, 213-241.

[11]Sripa B., Kaewkes S., Sithithaworn P., Mairiang E., Laha T., Smout M., Pairojkul C., Bhudhisawasdi V., Tesana S., Thinkamrop B., Bethony J.M., Loukas A., Brindley P.J. (2007) PLoS Med., 4, e201.

[12]Sukhdeo S.C., Sukhdeo M.V. (1990) Tissue Cell., 22, 39-50.

[13]Terenina N.B., Poddubnaya L.G., Tolstenkov O.O., Gustafsson M.K. (2009) Parasitol. Res., 104, 267-275. 